DRAFT VERSION MARCH 21, 2018

Preprint typeset using $\mathrm{AT}_{\mathrm{E} X} \mathrm{X}$ style emulateapj v. 01/23/15

\title{
MAGNETO-HYDRODYNAMICAL EFFECTS ON NUCLEAR DEFLAGRATION FRONTS IN TYPE IA SUPERNOVAE
}

\author{
Boyan Hristov ${ }^{1,2}$, David C. Collins ${ }^{2}$, Peter Hoeflich ${ }^{2}$, Charles A. Weatherford ${ }^{1}$, And Tiara R. Diamond ${ }^{3}$ \\ Draft version March 21, 2018
}

\begin{abstract}
This article presents the study of the effects of magnetic fields on non-distributed nuclear burning fronts as a possible solution to a fundamental problem for the thermonuclear explosion of a Chandrasekhar mass $\left(M_{\mathrm{Ch}}\right)$ white dwarf $(\mathrm{WD})$, the currently favored scenario for the majority of Type Ia SNe (SNe Ia). All existing 3D hydrodynamical simulations predict strong global mixing of the burning products due to Rayleigh-Taylor (RT) instabilities, which is in contradiction with observations. As a first step and to study the flame physics we present a set of computational magneto-hydrodynamic (MHD) models in rectangular flux tubes, resembling a small inner region of a WD. We consider initial magnetic fields up to $10^{12} \mathrm{G}$ of various orientations. We find an increasing suppression of RT instabilities starting at about $10^{9} \mathrm{G}$. The front speed tends to decrease with increasing magnitude up to about $10^{11} \mathrm{G}$. For even higher fields new small scale finger-like structures develop, which increase the burning speed by a factor of 3 to 4 above the field-free RT-dominated regime. We suggest that the new instability may provide sufficiently accelerated energy production during the distributed burning regime to go over the Chapman-Jougey limit and trigger a detonation. Finally we discuss the possible origins of high magnetic fields during the final stage of the progenitor evolution or the explosion.

Subject headings: instabilities, magnetic fields, magneto-hydrodynamics, turbulence, white dwarfs
\end{abstract}

\section{INTRODUCTION}

Type Ia supernovae (SNe Ia) are spectacular explosions at the end of the life of stars. Similar explosion energies, spectra, light curves (LC) and LC decline rates, $\Delta m_{15}$, established them as standard candles capable of measuring the Universe at the largest cosmological scales (Phillips 1993). Attempts to calibrate a brightness-decline relation began as early as Pskovskii (1977). Recently Li et al. (2003, 2011), Foley et al. $(\overline{2013})$ and Howell et al. (2006) identified the lax and the super-Chandrasekhar mass subclasses, which further complicates our understanding of these objects. A robust predictive model of SNe Ia first principles is in order.

It is widely accepted that a Type Ia supernova is a thermonuclear explosion resulting from a binary system, of which one star is necessarily a degenerate carbon-oxygen $(C-O)$ white dwarf (WD) (Hoyle \& Fowler 1960) near the Chandrasekhar mass $\left(M_{\mathrm{Ch}}\right)$. Current research considers various progenitor configurations and final outcomes. Depending on whether or not both stars are WDs, the progenitor system is called double degenerate (DD) or single degenerate (SD). In addition to progenitor configuration, proposed scenarios are distinguishable by the ignition mechanism and other characteristics. In the case of a DD progenitor system, a dynamical merger or a violent collision between the WDs is capable of releasing enough heat to trigger an ignition. This process can end up as a SN Ia, a highly magnetized WD (MWD), or an accretion-induced collapse (AIC) (Iben \& Tutukov 1984, Webbink 1984; Benz et al. 1990; Rasio \& Shapiro 1994 Segretain et al. 1997; Yoon et al. 2007; Wang et al. 2009b a| Lorén-Aguilar et al.|2009; Isern et al. 2011; Pak-

${ }^{1}$ Department of Physics, Florida A\&M University

${ }^{2}$ Department of Physics, Florida State University

NASA Goddard Space Flight Center, Greenbelt, MD 20771 mor et al. 2011). Another class of SNe Ia scenarios is the double detonation of a sub- $M_{\mathrm{Ch}} \mathrm{WD}$ with accretion from a helium $(\mathrm{He})$ companion. A detonation in the surface helium layer causes a secondary detonation in the core (Woosley et al. 1980; Nomoto 1982a, Livne 1990; Woosley \& Weaver 1994; Hoeflich \& Khokhlov 1996; Kromer et al. 2010; Woosley \& Kasen 2011). Finally there is the $M_{\mathrm{Ch}}$ explosions scenario, where the WD progenitor accretes material from a companion star and nuclear surface burning to $\mathrm{C} / \mathrm{O}$ leads to an increase of the WD mass. With increasing WD mass the electron gas in the central region becomes increasingly relativistic, which leads to faster compressional heat release, a raise of the central temperatures, and the triggering of a central $\mathrm{C} / \mathrm{O}$ deflagration front when the mass of the progenitor approaches $M_{\mathrm{Ch}}$. (Hoyle \& Fowler 1960, Sugimoto \& Nomoto 1980 , Nomoto 1982b; Hoeflich \& Stein 2002; Piersanti et al. 2003). It is likely that the dynamical merger, $M_{\mathrm{Ch}}$ explosion, and double-detonation channels all contribute to the SN Ia population because of the "stellar amnesia" effect (Hoeflich (2006), and references therein). This can happen in either a SD system, where the donor star is a main-sequence star, a red giant, etc., or in a DD system with another WD being the donor (Whelan \& Iben 1973: Piersanti et al. 2003).

In this paper, we will focus on the $M_{\mathrm{Ch}}$ explosion channel because of its consistency with a wide range of observations and their statistical properties. From observations we learn that the ejecta of a typical SNe Ia is made of chemical layers (e.g. Barbon et al. 1990; Kirshner et al. 1993; Hoeflich 1995; Fisher et al. 1997; Hoeflich et al. 2002, Marion et al. 2003, Stehle et al. 2005: Tanaka et al. 2011). The overall density structure shows only small deviations from spherical geometry based on the continuum polarization (Howell et al. 2001; Maund et al. 2010; Patat et al. 2012) and based on close to spherical 
supernovae remnants (Rest et al. 2005, Badenes et al. 2006, Fesen et al. 2007; Rest et al. 2008).

Despite the success of $M_{\mathrm{Ch}}$ explosions, there are serious problems related to mixing by the Rayleigh-Taylor (RT) instability during deflagration burning, prior to the phase of strong expansion. This instabilty results in strong large-scale mixing of the ejecta (Khokhlov 1995. Niemeyer \& Hillebrandt 1995; Livne 1999, Reinecke et al. 1999 Gamezo et al. 2003; Röpke et al. 2006; Plewa 2007). Although a layered chemical structure is partially restored during the detonation phase (Gamezo et al. 2005; Röpke et al. 2012), the predicted imprint of deflagration mixing is in contradiction to observations. Several observations of local SNe Ia strongly suggest that a process is necessary to partially suppress the dominant role of RT instabilities during the deflagration: (a) Direct imaging of the SNR s-Andromeda shows a large 'Ca-free' core, indicative of high-density burning and limited mixing (Fesen et al. 2007, 2015). (b) Spectral fits to observations of post-maximum spectra in normal-bright and subluminous SNe Ia are significantly degraded by injection of radioactive material into the S/Si layer (Figs. 12 and 14 in Hoeflich et al. 2002). (c) Flat-topped or stubby line profiles $1-2$ years after the explosion indicate stable isotopes remain near the center after the initial phase of burning (Hoeflich et al. 2004, Motohara et al. 2006 Maeda et al. 2010, 2011; Penney \& Hoeflich 2014; Stritzinger et al. 2014 Diamond et al. 2015). This is contrary to the mixing that would occur if RT full developed. Good agreement has been obtained with spherical models, whereas the flame physics is inherently multi-dimensional, which causes extensive mixing and strongly degrades individual fits and statistical properties. For a detailed discussion, see Höflich et al. (2006); Hoeflich et al. (2017).

Had there been a mechanism to suppress the RT instabilities in the early stages of the explosion, these discrepancies would be resolved. This requires a new piece of physics that has previously been left out by the current multi-dimensional models for the deflagration phase. High magnetic fields have been suggested as a solution (Hoeflich et al. 2004, Remming \& Khokhlov 2014, Hristov et al. 2016). Several points support this suggestion. (a) From both theory and simulation, tension in the magnetic field suppresses RT instabilities parallel to the field and also secondary instabilities perpendicular to the field (Chandrasekhar 1961; Stone \& Gardiner 2007a b). (b) Magnetic surface fields in a wide range of strengths have been observed in WDs (Liebert et al. 2003, Kawka et al. 2007; Giammichele et al. 2012; Kepler et al.| 2013; Sion et al. 2014; Ferrario et al. 2015, Kepler et al. 2016). While these observations indicate small fields, stronger fields are possible both within the core of the WD and at shorter time scales before the explosion (see Section 4). (c) Positron transport effects on light curves and spectral line profiles are expected at late times for explosions without magnetic fields (e.g. Milne et al. 1999 ; Penney \& Hoeflich 2014). However, these were not seen in SN2011fe and SN2017j, which were observed for some three years past maximum light (Kerzendorf et al. 2017. Yang et al. 2017). (d) Penney \& Hoeflich (2014) used late-time near infrared line profiles to estimate magnetic fields. Though the number of SNe Ia with late-time IR spectra is small, recent observations strongly suggest that initial high magnetic fields with strengths, $B>10^{6} \mathrm{G}$ are
TABLE 1

Model (RUn) NAMES, CHARACTERIZED By THE SiZE AND ORIENTATION OF THE INITIAL $B$-FIELD.

\begin{tabular}{|c|c|c|c|}
\hline \multirow{2}{*}{$\begin{array}{l}\text { Initial Magnitude } \\
\text { (Gauss) }\end{array}$} & \multicolumn{3}{|c|}{ Initial Direction } \\
\hline & $\mathbf{B} \| \widehat{\mathbf{y}}$ & $\mathbf{B} \| \widehat{\mathbf{y z}}$ & $\mathbf{B} \| \widehat{\mathbf{z}}$ \\
\hline $1.4 \times 10^{12}$ & Y12 & YZ12 & $\mathrm{Z} 12$ \\
\hline $1.4 \times 10^{11}$ & Y11 & YZ11 & Z11 \\
\hline $1.4 \times 10^{10}$ & Y10 & YZ10 & Z10 \\
\hline $3.5 \times 10^{9}$ & Y9 & YZ9 & Z9 \\
\hline $3.5 \times 10^{8}$ & Y8 & YZ8 & Z8 \\
\hline 0 & $B=0$ & $(B=0)$ & $(B=0)$ \\
\hline
\end{tabular}

Note. - The front propagates in the $\widehat{\mathbf{z}}$ direction opposite to the gravitational acceleration, $\mathrm{g}$. Figures 1 and 2 follow the same layout

common (Hoeflich et al. 2004, Penney \& Hoeflich 2014 Diamond et al. 2015), even assuming fields on small-scales comparable to the pressure scale height of the WD.

The effects of magnetic fields on the nuclear burning in $\mathrm{SNe}$ Ia and in a stellar context have been studied in previous works. Potekhin \& Chabrier (2012) investigate the effects of magnetic fields on non-resonant thermonuclear reactions in the interiors of $C-O$ WDs. Kutsuna \& Shigeyama (2012) study laminar flames in magnetic fields at densities that are typical for a $M_{\mathrm{Ch}} \mathrm{WD}$. Ji et al. (2013) simulated a MWDs merger on an axisymmetric cylindrical (2D) domain, followed by Zhu et al. (2015) in full 3D, who obtained final fields of $>10^{10} \mathrm{G}$. Ghezzi et al. (2001, 2004) employ 1D semi-analytic flame models and find that the flame speed is affected by suppression of RT instabilities and additionally study the asymmetries of a deflagration front in a WD with a large-scale dipole field.

Theories about the origin of WD magnetic fields and in support of amplification of the fields during different stages of the star or the binary system evolution are discussed in the final section.

The article is organized in the following way. We describe our method in Section 2 starting with arguments about our choices of magnetic field strength and topology, the initial conditions and boundary conditions, and the regime of burning. The base MHD code, Enzo, is outlined in Section 2.2 and the newly added nuclear reaction model in Section 2.3. Our results are presented in Section 3. In Section 4, we discuss the results, as well as future work and current dynamo theories. The latter is not part of this study but is important to justify the use of high magnetic fields in the central region of the WD. All equations are written in the $c g s$ system of units. Other units are used in plots and throughout the text on occasion.

\section{METHOD}

To investigate the effects of magnetic fields on the nuclear burning we ran 16 models identical in all but the magnitude and direction of the initial magnetic field. These runs are listed in Table1. One model has no magnetic field and the rest are initialized with a uniform magnetic field covering 5 orders of magnitude between $10^{8} \mathrm{G}$ to $10^{12} \mathrm{G}$, and vary between 3 directions; perpendicular to the WD radius, parallel to the WD radius and at $45^{\circ}$, labeled respectively $\widehat{\mathbf{y}}, \widehat{\mathbf{z}}$, and $\widehat{\mathbf{y z}}$. The main 

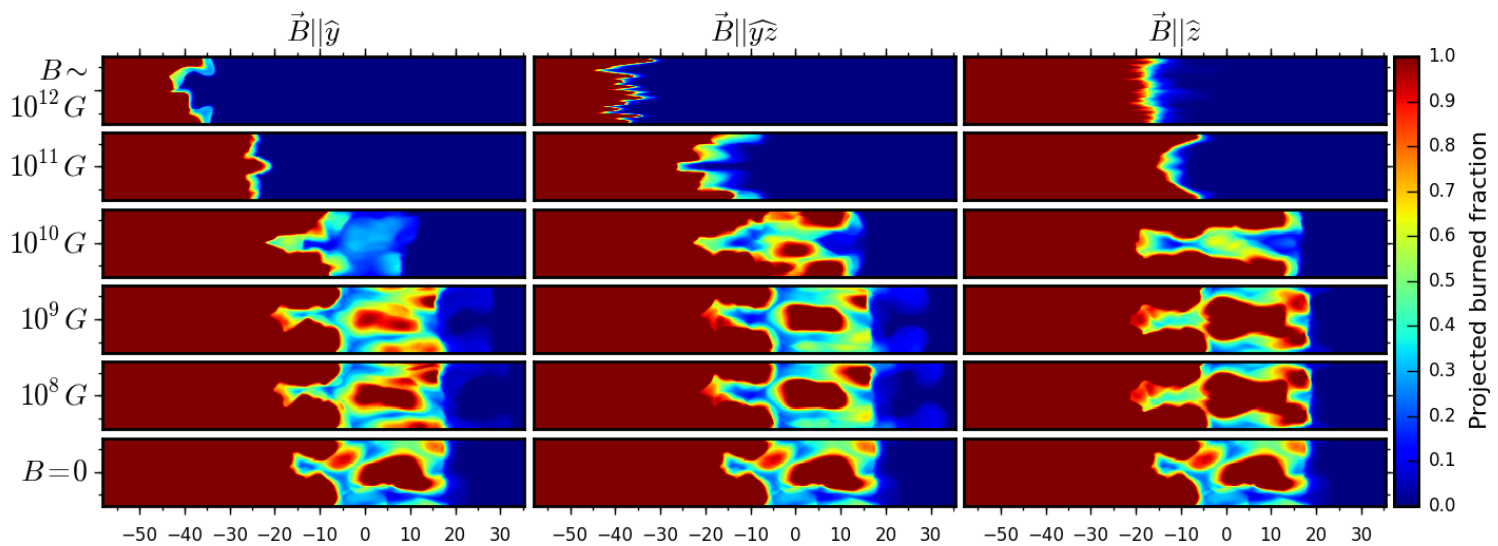

(a) Fuel molar fraction at $t=0.6 \mathrm{~s}$ projected along the $y$-axis.

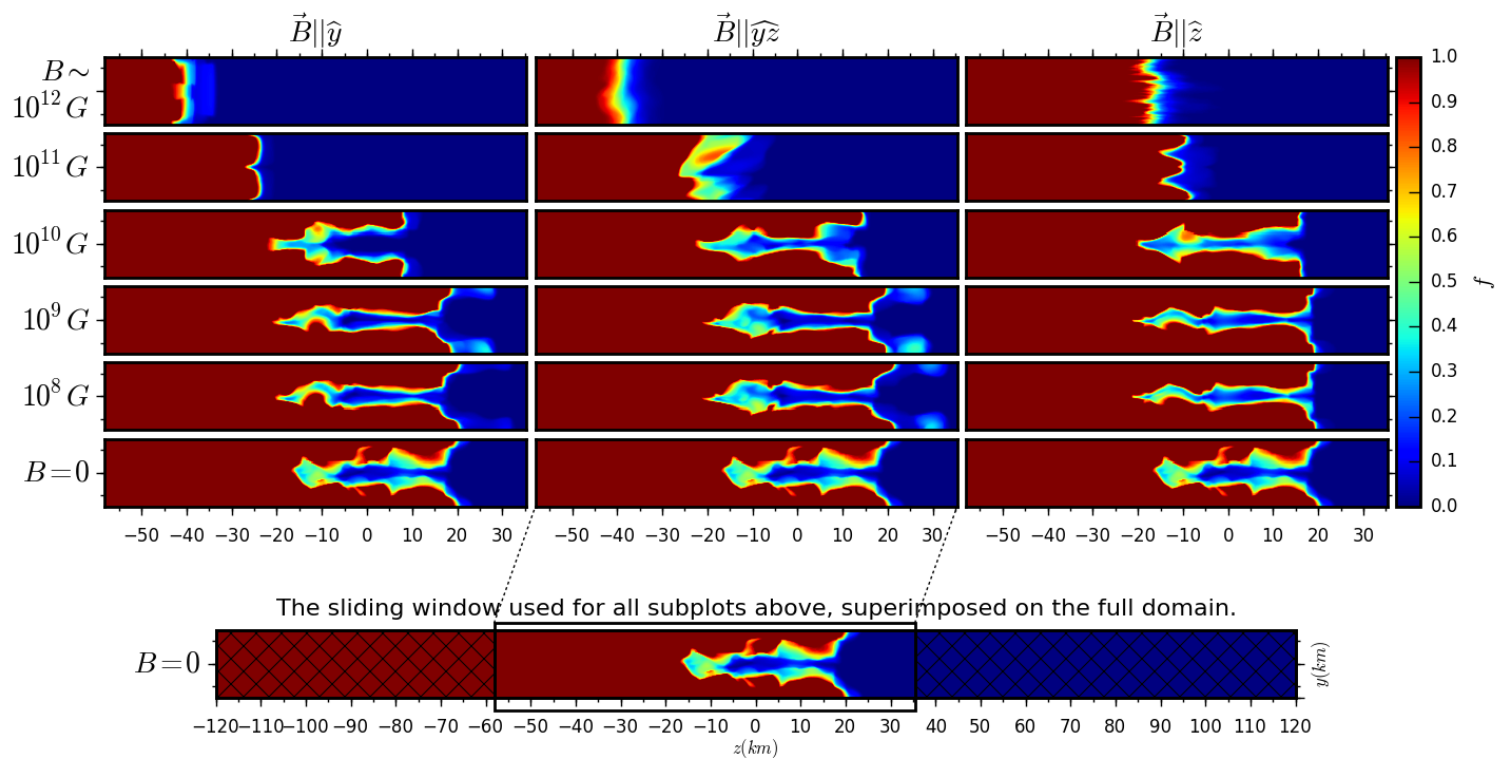

(b) Fuel molar fraction at $t=0.6 \mathrm{~s}$ projected along the $x$-axis.

FIG. 1. - Effects of $B$ on the nuclear burning front. At low or no field (bottom row and up) one there is little difference in the behavior. Turbulence dominates and cusps of fuel appear. For models with $B_{0}=10^{10} \mathrm{G}$ one can see some stabilization effects of the front, most notably in Y10. For models Y11 and Y12, where the field is in the plane of the front, the front becomes laminar. For models YZ12 and Z12, the strong-field models with a component of the field in the direction of the propoagation, we see another change in the burning properties. Smaller modes along $z$ appear where the burning is accelerated. The modes are stabilized by the strong magnetic field. As a result, the fuel burns at a higer rate. We suggest that this may be a mechanism to accelerate the front over the Chapman-Jougey limit and trigger a transition to detonation. See also Fig. 2

results can be sen in Figures 1 and 2, Figure 11 shows the burned fraction for projections along $\hat{y}$ (panel a) and $\hat{x}$ (panel b). Columns denote initial field orientation, and rows denote initial field strength. Figure 2 shows the burning rate for each snapshot. These figures are at $t=0.6 \mathrm{~s}$. The bottom plot shows the position of the burning front in the computational domain. These will be discussed further in Section 3. To ensure the results are insensitive to the boundary, an additional simulation was executed based on model YZ10, but with twice the length of the domain. This longer domain did not change the results. We note that the width of the domain imposes a maximum length of the possible modes, which in turn allows for the existence of a sufficient magnetic field suppressing all linear perturbations (see, for example, discussion in Stone \& Gardiner 2007b).

\subsection{White dwarf setup}

Our work is in the context of a MWD undergoing a $M_{\mathrm{Ch}}$-scenario explosion. Model parameters were chosen to resemble the physical conditions of a WD after the onset of the deflagration stage, long enough for RT instabilities to set up and while the burning is still in the non-distributed regime.

As this is our first exploration of the impact of magnetic fields on WD, we use a simplified picture in order to understand the physics of the burning front in the presence of a magnetic field. We use rectangular tubes with an initially uniform magnetic field, embedded in the inner region of a WD (Fig. 3.) We solve the models numerically using full 3D ideal MHD with non-distributed nuclear burning.

\subsubsection{Magnetic field magnitude}

We consider $B$ magnitudes up to $10^{12} \mathrm{G}$, which is close to those for a virialized WD at $10^{13}-10^{14} \mathrm{G}$ (Alvear \begin{tabular}{l|l|l|l|}
\hline Terrero et al. 2015, Franzon \& Schramm 2017). As an
\end{tabular} 


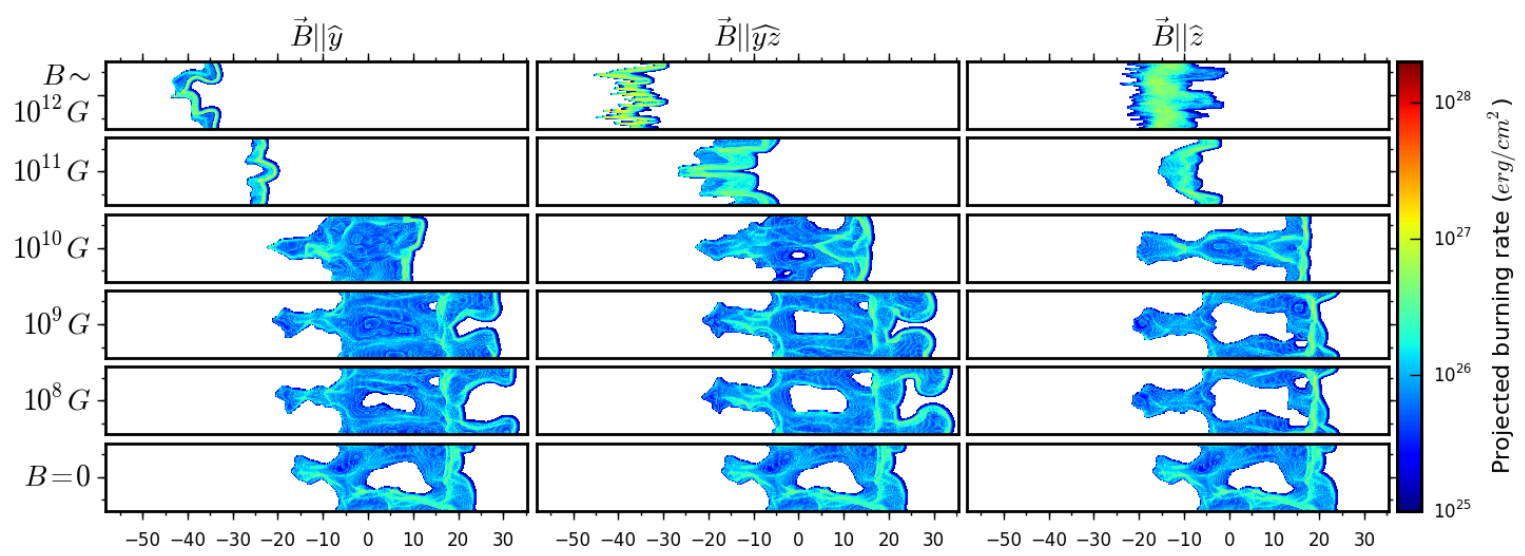

(a) Energy production density rate projected along the $y$-axis.

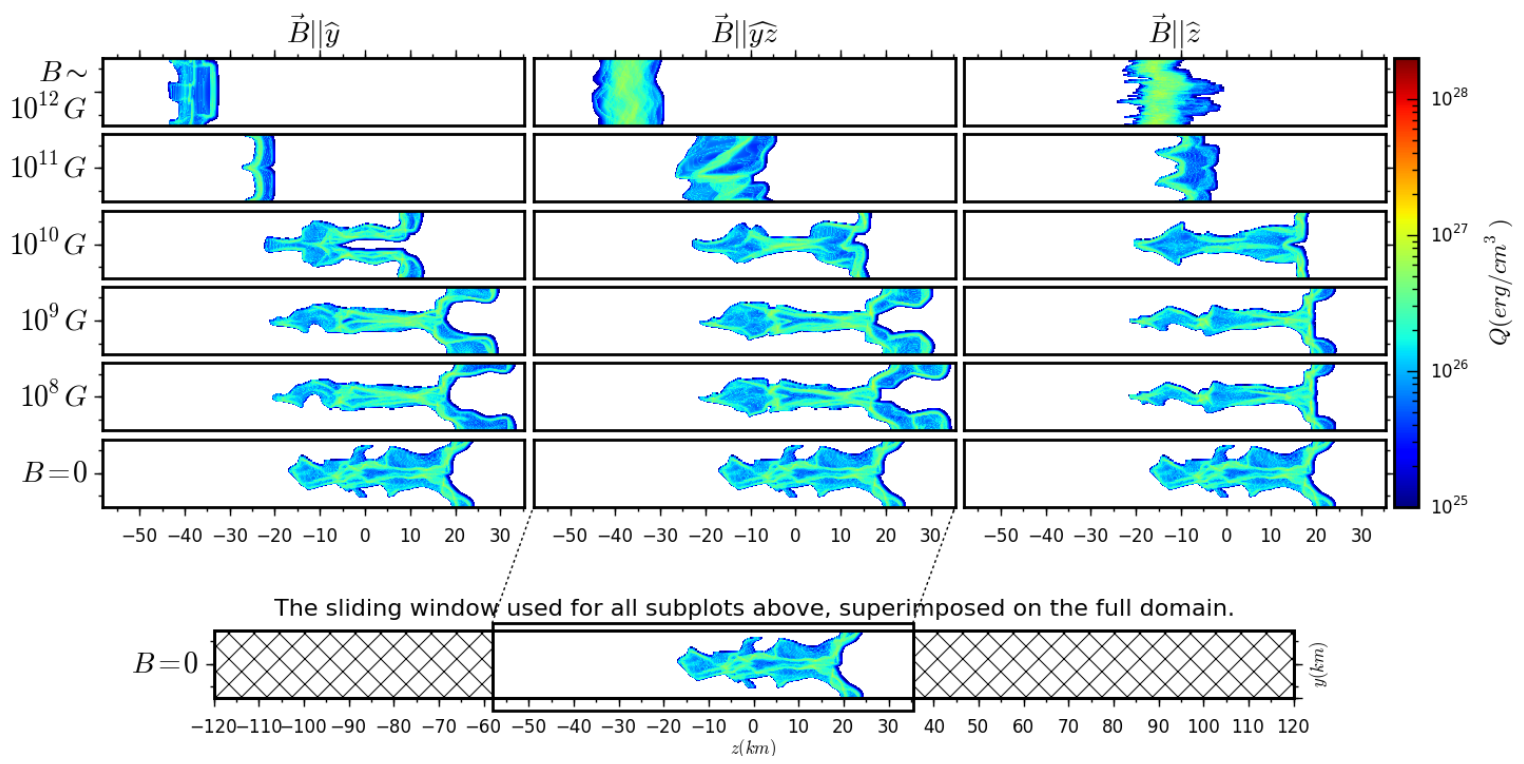

(b) Energy production density rate projected along the $x$-axis.

FIG. 2.- Energy production density rate projections at $t=0.6 \mathrm{~s}$. See also Fig. 1 .

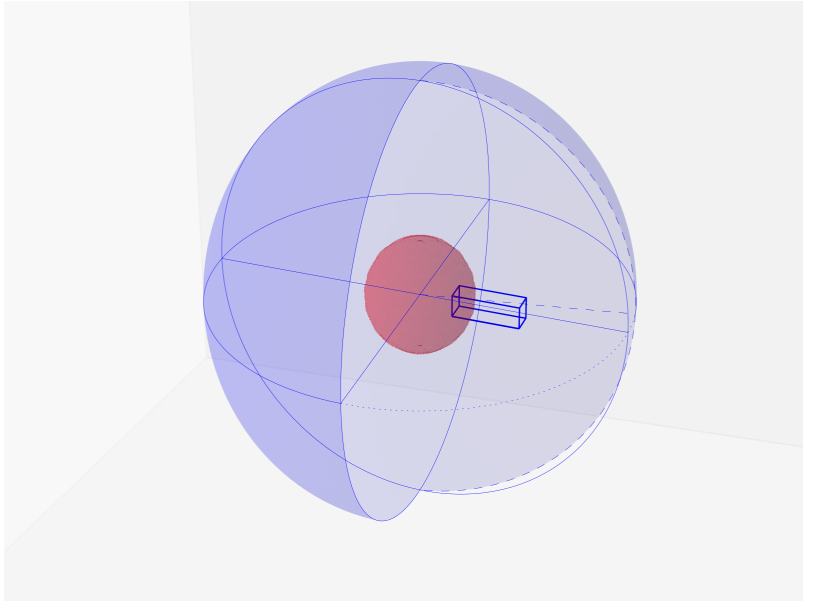

Fig. 3.- A sketch of the location of our flux tube within the white dwarf. This is close to the center, in the regime of nondistributed burning. See Section 2.1.3 for more details.

order-of-magnitude estimate of the field strength, we can equate magnetic energy with gravitational binding energy. For a density of $\rho=10^{8} \mathrm{~g} / \mathrm{cm}^{3}$ and a radius of $1,600 \mathrm{~km}$, using a binding energy of $G M^{2} / R$ and magnetic energy of $B^{2} R^{3}$, one finds a field strength of $4 \times 10^{12} \mathrm{G}$.

MWDs are commonly observed in the magnitude range $10^{3}-10^{9}$ G (Liebert et al. 2003, Kawka et al. 2007, Giammichele et al. 2012; Kepler et al. 2013; Sion et al. 2014; Kepler et al. 2016), where the observations suggest a real cutoff at the upper bound and a sensitivity-limited lower bound (Ferrario et al. 2015). However, these observations are of non-exploding WDs, and the observed magnetic field magnitudes are only on the surface of the WD. Here, while we want to model a region close to the center and after a central ignition has occurred, which is a regime not probed by these observations. Small-scale dynamo theory, as well as virial analysis, suggest that fields as large as $10^{12} \mathrm{G}$ are possible in the conditions expected before the explosion. This will be discussed further in Section 4.2

\subsubsection{Field morphology considerations}

Field morphology can become very complex, as predicted by theory and observations (see Reinsch et al. 2005, and discussion therein). It is also uncertain whether the field is a dipole (or a multipole) on a large scale or turbulent, or if there is a combination of fields 
at different scales. We try to accommodate both cases by considering the order of the typical turbulent eddy, $l_{\text {turb }} \sim 100 \mathrm{~km}$, established in Hoeflich \& Stein (2002). Furthermore, the domain needs to be long enough to allow the advancement of the burning front for about a second, as well as to make the boundary effects remote enough. With this in mind we made our computational domain $240 \times 15 \times 15 \mathrm{~km}(1024 \times 64 \times 64$ computational zones, see also section 2.1.4 .

\subsubsection{Initial and boundary conditions}

Immediately after a central ignition, RT instabilities would not develop because close to the center of the WD the gravitational acceleration, $\mathrm{g} \simeq 0$. We need to pick a later time when the burning front becomes RT-unstable. In addition we are interested in the early evolution of the WD, because plumes created early on have the most time to rise and therefore would create the most mixing. Such conditions are realized in a model in Hoeflich (2006) at time $t=0.1 \mathrm{~s}$ when the burning front has reached about 1,700 km from the center of the WD. At that time and location $\mathrm{g}$ and $\rho$ change little within the span of the domain, $L_{z}$, so we initialize them with constants $\mathrm{g}=$ $1.9 \times 10^{9} \mathrm{~cm} / \mathrm{s}^{2}$ and $\rho_{0}=10^{8} \mathrm{~g} / \mathrm{cm}^{3}$. Additionally we keep g constant in time.

We place the burning front at $10 \mathrm{~km}$ from the bottom and perturb its plane, so the initial front surface is described by $\left.\zeta(x, y)\right|_{t=0}=A_{0}\left(\cos \left(k_{0} x\right)\right)+\cos \left(k_{0} y\right)$, where $k_{0}=2 \pi / \lambda_{0}$. For all simulations, $A_{0}=5 \times 10^{4} \mathrm{~cm}$ and $\lambda_{0}=4.8 \times 10^{5} \mathrm{~cm}$. We initialize the region below the burning front with completely burned material and the one above with unburned fuel. Both regions have the same uniform initial density of $\rho_{0}=10^{8} \mathrm{~g} / \mathrm{cm}^{3}$. The initial specific internal energy is also uniform yet different on both sides of the burning front, $3.3 \times 10^{18}$ and $0.4 \times 10^{18} \mathrm{erg} / \mathrm{g}$ in the burned and unburned regions respectively, corresponding to initial pressures of $8.25 \times 10^{26}$ and $10^{26} \mathrm{dyn} / \mathrm{cm}^{2}$. The initial velocity is zero everywhere.

\subsubsection{Non-distributed regime of burning}

At the burning front, with temperatures of $T \gtrsim$ $10^{9.5} \mathrm{~K}$ and densities of $\rho_{0} \approx 10^{8} \mathrm{~g} / \mathrm{cm}^{3}$, the laminar front thickness drops to the order of centimeters: $l_{\text {flame }} \sim 10^{-2}-10 \mathrm{~cm}$ (Timmes \& Woosley 1992). This is much smaller than other length scales that an attempt to capture the details of the burning front would be a formidable computational task. As a consequence, and with the burning being complete in this regime, we cannot resolve any intermediate isotopes but only fuel and burning products. We need a grid resolution, $\Delta x$, small enough to reproduce accurate MHD features, but much coarser that the front scale length, $\Delta x \gg l_{\text {flame }}$, and we use one similar to the resolution in Khokhlov (1995) (see 2.1.2

We chose a laminar front speed of $10 \mathrm{~km} / \mathrm{s}$ also after Khokhlov (1995), which is slightly higher than the velocity in Timmes \& Woosley (1992). Additionally we used a quasi-1D model to measure the numerical laminar front thickness and speed. This model is similar to the B0 model, except that there is no perturbation of the initial burning front surface, as well as there is no gradient in initial pressure $\left(10^{26} \mathrm{dyn} / \mathrm{cm}^{2}\right.$ everywhere $)$. The re- sults from this simulation confirmed that the model laminar velocity is $10 \mathrm{~km} / \mathrm{s}$. Furthermore the flame thickness in our simulation is stretched over 4-5 computational zones (about $1 \mathrm{~km}$ ), which is typical for shock-capturing schemes.

\subsection{Code}

For the data presented in this paper, we solve the ideal MHD equations with nuclear burning using the adaptive mesh refinement (AMR) code Enzo (Bryan et al. 2014) extended to MHD by Collins et al. (2010). The nuclear burning is a new addition to the code, and described in Section 2.3. The MHD solver uses the hyperbolic solver of Li et al. (2008), the isothermal HLLD Riemann solver developed by Mignone (2007), and the Constrained Transport (CT) method of Gardiner \& Stone (2005). The simulations presented here were run with fixed resolution. Enzo has been used in a diverse array of astrophysical settings, including star formation (Abel et al. 2002, Collins et al. 2012), supersonic turbulence (Kritsuk et al. 2007), x-ray gas in clusters, (Bryan \& Norman 1998), and large-scale structure (Bryan et al. 1999, O Shea et al. 2015), and the epoc of reionization (Norman et al. 2015), among others. For simplicity, all simulations are run with fixed resolution

The MHD equations with burning are as follows:

$$
\begin{gathered}
\frac{\partial \rho}{\partial t}+\nabla \cdot(\rho \mathbf{v})=0 \\
\frac{\partial \rho \mathbf{v}}{\partial t}+\nabla \cdot\left(\rho \mathbf{v} \mathbf{v}+\mathbf{I} P-\frac{\mathbf{B B}}{8 \pi}\right)=-\rho \mathbf{g} \\
\frac{\partial E}{\partial t}+\nabla \cdot\left[(E+P) \mathbf{v}-\frac{\mathbf{B}(\mathbf{B} \cdot \mathbf{v})}{4 \pi}\right]=-\rho \mathbf{v} \cdot \mathbf{g}+\dot{Q} \\
\frac{\partial \mathbf{B}}{\partial t}+\nabla \times(\mathbf{v} \times \mathbf{B})=0
\end{gathered}
$$

where $\mathbf{v v}$ and $\mathbf{B B}$ are the velocity and the magnetic field outer products and $\rho, \mathbf{g}$, and $\dot{Q}$ are the density, the gravitational acceleration, and the energy production rate from the nuclear burning. The total energy density is equal to the total of the thermal, the kinetic and the magnetic energy densities,

$$
E=e+\frac{\rho v^{2}}{2}+\frac{B^{2}}{8 \pi}
$$

and

$$
P=p+\frac{B^{2}}{8 \pi}
$$

is the total pressure, which is the thermal plus the magnetic pressure.

A particular realization of a degenerate relativistic equation of state (EOS) from Hoeflich (2006) is shown in Fig. 4. Considering the right two panels, one can assume that at temperatures $T \gtrsim 10^{9.5} \mathrm{~K}$ and at densities in the range $\rho=10^{7}-10^{8} \mathrm{~g} / \mathrm{cm}^{3}$, the adiabatic index $\gamma=$ const is a reasonable approximation. Hence we close the system with the equations of state for an ideal gas,

$$
e=\frac{p}{\gamma-1}
$$


where the adiabatic index $\gamma=1.4$ for these simulations.

\subsection{Burning}

We model the nuclear burning and flame propagation after Khokhlov (1995), which takes advantage of the nondistributed regime in a couple of ways. Firstly it takes a grid resolution much coarser than the flame scale length, as discussed in Section 2.1.4. Then it approximates the real nuclear network with one that has only two elements:

$$
\text { fuel } \rightarrow \text { product }+\mathcal{Q}
$$

where $\mathcal{Q}$ is the specific energy produced from burning. In our simulations the fuel consists of equal amounts by number of ${ }^{12} \mathrm{C}$ and ${ }^{16} \mathrm{O}$ with a mean atomic weight $\mathcal{A}_{\text {fuel }} \approx 14$, and the product is ${ }^{56} N i$. We define the burned molar fraction, simply referred as burned fraction, $f$, as

$$
f=\frac{\mathcal{Y}_{\text {prod }}}{\mathcal{Y}_{\text {fuel }}+\mathcal{Y}_{\text {prod }}}
$$

where $\mathcal{Y}_{i}$ are the abundances. Note that $f \in[0,1]$, where $f=0$ corresponds to pure fuel, and $f=1$ means pure product. A nuclear deflagrating flame ignites unburned fuel by heating through electron conduction. The model flame spreads by diffusing the burned fraction, $f$ :

$$
\frac{\partial f}{\partial t}+\mathbf{v} \cdot \nabla f=K \nabla^{2} f+R(f) .
$$

Here $K$ approximates the diffusion of burning material, thus energy to ignite the up-stream fuel. The instantaneous burning rate is then denoted by $R$. The front becomes stretched over a few grid cells, as is typical for shock-capturing schemes. The laminar speed velocity should be proportional to $\sqrt{K R}$. We chose a value of $10 \mathrm{~km} / \mathrm{s}$, which we confirmed in a $1 \mathrm{D}$ run. According to Remming \& Khokhlov (2016), the laminar front velocity depends on the magnetic field strength and direction in addition to the fuel density. However in our regime the difference the authors had calculated is about $3 \%$, allowing us to assume constant values for $K$ and $R$. Additionally we turn $R$ on and off locally depending on the burned fraction:

$$
R=R(f)=\left\{\begin{array}{l}
R_{0}, \quad \text { if } f_{0} \leq f \leq 1 \\
0, \quad \text { if } 0 \leq f<f_{0}
\end{array}\right.
$$

and $f_{0}=0.3$ as in Khokhlov (1995).

This delays the ignition of the fuel locally, preventing the entire domain from been ignited in the first few time steps by non-physical numerical waves traveling faster than the physical laminar flame. Finally, the burning energy is calculated as:

$$
\dot{Q}=\mathcal{Q} \frac{d \rho_{\text {prod }}}{d t}
$$

\section{RESULTS}

As described in Section 2, Figures 1 and 2 show the effect of magnetic field on the propagation of nuclear burning in our simulations. There is a clear impact of the magnetic field on the speed and morphology of the front in the strongest field simulations, but one can see that all of the tested fields leave some imprint on the burning front regardless of the initial magnitude or direction. At low magnetic field, $\left(B \lesssim 10^{9} \mathrm{G}\right.$, and including no field at all, bottom row in those figures), the relative initial pressure (thus initial density) gradient is opposite to gravity, making the flow RT-unstable. Large modes grow faster, as expected, and they would continue to rise. The flame morphology becomes increasingly complex. The burning front develops chaotic structure and is dominated by turbulence. Small modes grow slower and are washed out by the flow shear or the diffusion by which the laminar front advances (Khokhlov 1995, see also eqn. 10) The effective front width is $\sim 50-70 \mathrm{~km}$. Some pockets of fuel are formed.

Going up in magnitude to $10^{10} \mathrm{G}$, we can recognize a trend of the effective width of the burning front shrinking. This is most pronounced in the case of a transverse field. Turbulence is still prominent, and the front is comparable to that of the lower $B$ cases.

At the highest magnetic field strengths, stabilization effects are well manifested. The chaotic behavior is suppressed, and the front structure is closer to laminar. The effective front width is now only on the order of $10 \mathrm{~km}$. For models Y11, YZ11 and Y12 the flame front closely follows the field lines.

For models Z12 and YZ12 we identify another change in the behavior. The front becomes jagged with smaller peaks oriented along $z$. In YZ12 these are seen only in the $y$-projection. This behavior is mirrored in the front velocity and energy production, shown in Figures 5 and 6, respectively. In Figure 5, the black line shows the velocity of the fiducial un-magnetized run. After an initial transient phase, as the instability sets in prior to $t=0.3$ $\mathrm{s}$, it is clearly seen that the moderately magnetized runs (blue lines at $10^{10} \mathrm{G}$ and purple lines at $10^{11} \mathrm{G}$ ) move slower due to the decreased surface area of the front. This decrease is also seen in the energy production rate, which can be seen as a proxy for the surface area, see eqn. 11. In these runs, the instability is suppressed as expected by perturbation analysis, discussed in Section 3.1. For the largest field runs that also contain a component along the propagation direction, however, the behavior is somewhat counterintuitive. These runs show a significant increase in the front velocity over all other runs. This is due to suppression of secondary KelvinHelmholtz instability, see Section 3.2

\subsection{Linear Stability Analysis}

The MHD equations (1, 4) can be perturbed about an interface subject to an acceleration, and the growth rates calculated, as is done in standard Rayleigh-Taylor or Kelvin-Helmholtz instability studies. The magnetic field can exert tension in the front, and tend to stabilize the flow. Details are left to Appendix C, but we will discuss the salient results here. The analysis assumes that the magnetic field is either parallel or perpendicular to the gravity, $\mathbf{g}$ and the position of the front is assumed to behave as $\exp \left(i k_{x} x+i k_{y} y+\Omega t\right)$. Here, a negative $\Omega^{2}$ indicates a stable mode. The dispersion relation for the field perpendicular to $\mathbf{g}$, presented on the bottom panel of Fig. 7, shows that the only simulations with some stable modes in the linear regime are Y11, YZ11 and Y12. All other orientations, at all modes, remain unstable. This explains the stark contrast between the 

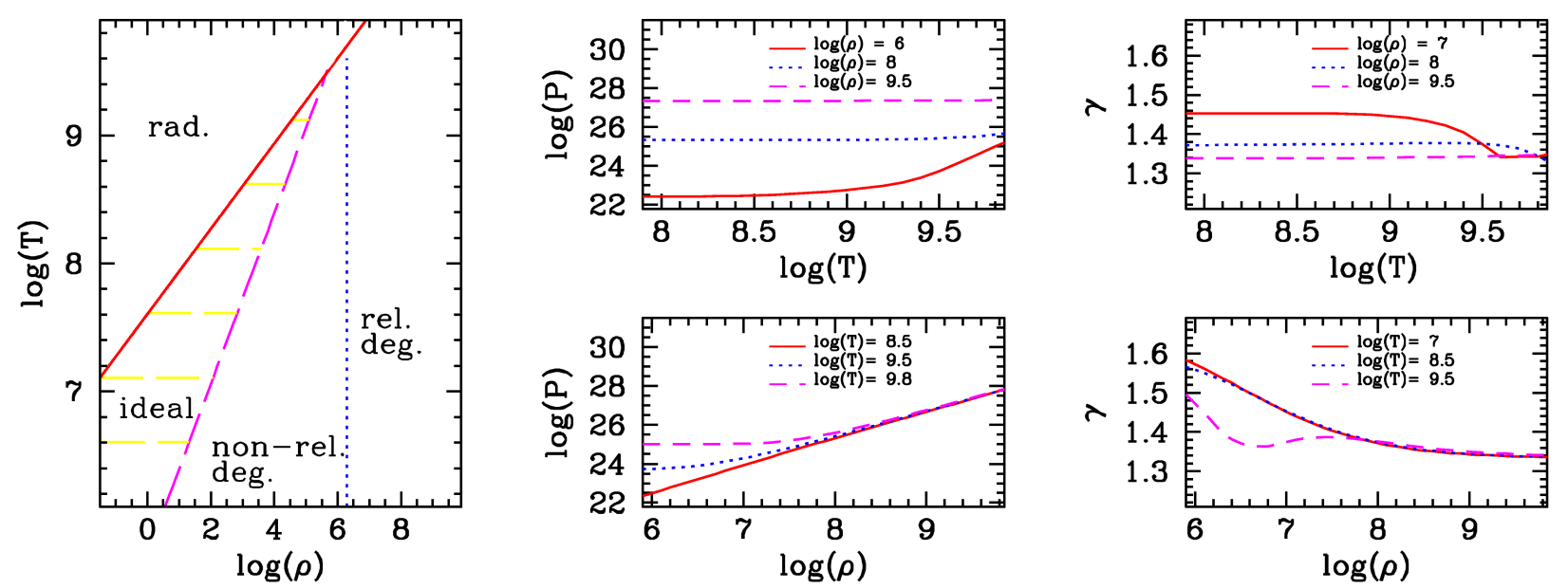

FIG. 4. - EOS of a WD (Hoeflich 2006). The two right panels show that $\gamma$ depends relatively weakly on $T$ and on $\rho$ in the non-distributed regime of burning $\left(T \geq 10^{8.5} \mathrm{~K}\right.$ and $\left.\rho \sim 10^{8} \mathrm{~g} / \mathrm{cm}^{3}\right)$, hence it can be taken to be a constant.

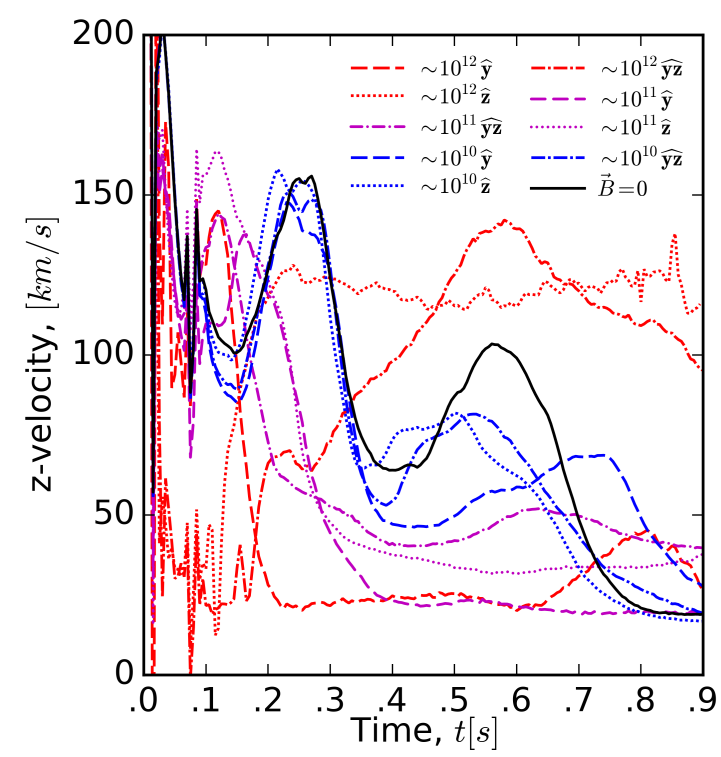

FIG. 5. - Flame speed in the frame comoving with the bulk flow. The $10^{8} \mathrm{G}$ and $10^{9} \mathrm{G}$ profiles are omitted for clarity. For most simulations, the velocity is decreased over the non-magnetized case by the reduction of the surface area of the front. The Z12 and YZ12 velocities are larger than any other case as these regimes have new kind of instability as discussed in Section 3.2

top two rows and the other four in Figures 1 and 2 . Having stable modes above a certain magnetic field strength is an effect of the finite domain size, as mentioned in the beginning of Section 2 ,

When the field is parallel to the gravity, the top panel of Fig. 7 shows no stable linear modes regardless of the field strength. This not a limitation of the domain size or the resolutiuon but works for all wave numbers as proved in Chandrasekhar (1961). We also note that the growth rate is monotonic with respect to the size of the perturbation, with the smallest modes growing fastest. However any of the calculated growth rates (considering all models, including $B=0$ ) do not excede $10^{-4}$, which means that in this case the duration of the problem, $\sim 1 \mathrm{~s}$ is too short for any linear perturbations to grow.

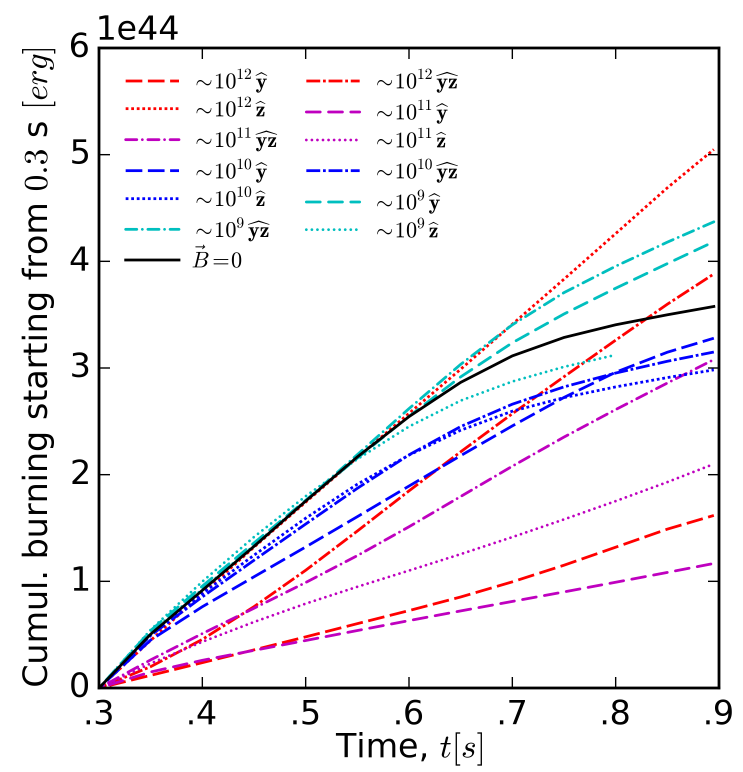

FIG. 6.- Cumulative burning energy, integrated from $t=0.3 \mathrm{~s}$. The $10^{8} \mathrm{G}$ profile is not shown for clarity. In order to remove the effects of the initial conditions, seen in Fig. 5 we integrate the burning rates starting right after the burst. We note a trend for the rates to drop with increasing field magnitude up to $10^{11} \mathrm{G}$. Beyond that models Z12 and YZ12 show the steepest slopes as well as steady state processes.

\subsection{Nonlinear Growth}

The linear stability is useful for guiding intuition, but the flow in question is no longer in the linear regime.

In order to quantify this impression we applied a Fourier transform to the $95 \%$ iso-surface of the burned fraction in all $10^{11}$ and $10^{12}$ runs, shown in Fig. 8 . This is a good method for the YZ12 and Z12 models, since the said iso-surface is a single-valued function, $\zeta(x, y)$. When the front has multiple points for the same $(x, y)$ we take the rightmost one. Most profiles have the same power for the medium modes in the range $L / \lambda=8-15$ zones. However these are dominated by the larger modes $(L / \lambda=1-6$ zones) and appear as noise. The Z12 spectrum, on the other hand, shows suppressed large modes to the same level of power as the medium modes. There- 

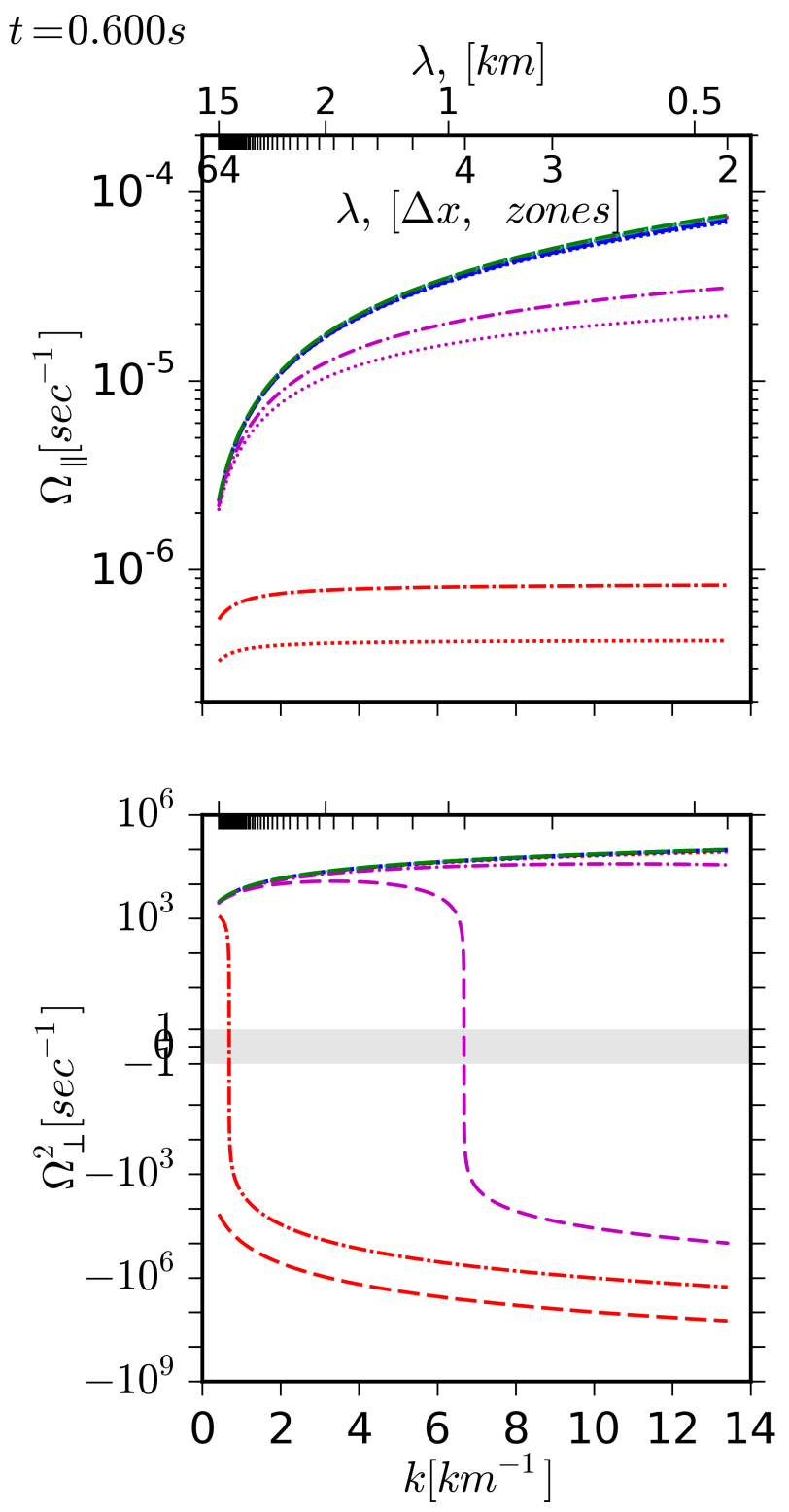

FIG. 7.- Growth rate of perturbation modes. See legend on Fig. 6 Negative values indicate stable modes. The theoretical dispersion relations are used for magnetic fields parallel (Eqs. C1 C3), and perpendicular (Eq. C4) to $\mathrm{g}$ at $t=0.6 \mathrm{~s}$. The wavelength range starts from 2 computational cells up to the entire domain width. For the perpendicular cases (bottom panel) we see that stable modes can be expected only in three simulations, whereas all modes are stable in the Y12 run. This is a rough agreement with the flows shown in Fig. 1 especially in the $\widehat{\mathbf{y}}$ models. We also see stable surfaces along the magnetic field in the YZ11 run. In the parallel cases (top panel) none of the simulations show stable modes. and the smallest modes grow the fastest. However the growth rate is suppressed so much that no growth will occur for the duration of the problem, $\sim 1 \mathrm{~s}$.

fore we cannot consider the latter as noise.

Furthermore the side walls of these instabilties become almost parallel to $\widehat{\mathbf{z}}$, where the sinking fuel and the rising burned material create shear at the boundary. These conditions are right for developing Kelvin-Helmholtz instabilities, but the magnetic field is now almost parallel to the shear interface, adding surface tension and making the flow laterally stable. The condition for Kelvin-

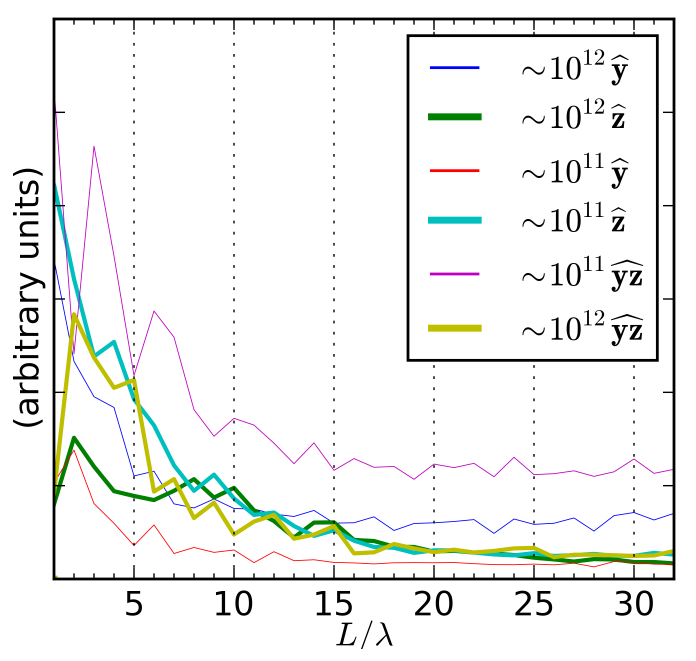

Fig. 8.- Fourier transformation of the flame as a function of $x$ and $y$ for the high-field simulations. All profiles are normalized so they have maximum 1 , in order to emphasize the distribution of power within each simulation, rather than comparing powers between simulations. The Z12 spectrum shows stronger features for $L / \lambda=8-15$ compared to the rest. This is due to the lateral magnetic support against secondary instabilities once perturbations become elongated. Additionally all profiles become flat for $L / \lambda \geq 16$ with little power in that region, i.e. only modes larger than two computational cells are prominent. This can be attributed to competition of advection vs. diffusion, but could be a resolution effect as well and needs to be studied further.

Helmholtz stability, derived in the stability analysis in the Appendix (in Eq. C5) is visualized in a 1-zone slice in Fig. 9. It holds for all zone boundaries parallel to $\widehat{\mathbf{z}}$. Somewhat similar effects to the RT fingers were observed by Stone \& Gardiner (2007a b) in lateral direction caused by magnetic fields perpendicular to the gravity. Most importantly they consider non-recative fliuids and observe bubble growth poportional to $t^{2}$. In our models burning is expected to limit this growth in all regimes. Specifically in Z12 and YZ12 models the height of the burning zone, once established, remains about the same through the simlulation.

We also observe that all spectra become flat for $L / \lambda>$ 15 with the least power in those modes. Since the lateral advection is suppressed we could attribute this effect to diffusion, however it could be something else, including numerical.

Fig. 9 shows fingers of fuel with a width comparable to the flame laminar thickness, 3-4 zones. Poludnenko \& Oran (2011) and Hicks (2015) found that when the radius of curvature at the bottom of the fingers becomes on the order of the laminar flame thickness or less, the fuel burns at an accelerated rate. The plots in Figures 5 and 6 confirm this. We note that the the burning rates and the front speeds tend to drop with magnitude and are smallest at about $10^{11} \mathrm{G}$ due to the decrease of the front surface, but in the Z12 and YZ12 models the profiles become steeper again.

\section{DISCUSSION}

We show that a magnetic field in the WD deflagration regime has an effect on nuclear burning for magnitudes $B \gtrsim 10^{10} \mathrm{G}$. At medium to high magnitudes we observe a front that is more organized compared to low magnetic field strength or no field at all. The burning 


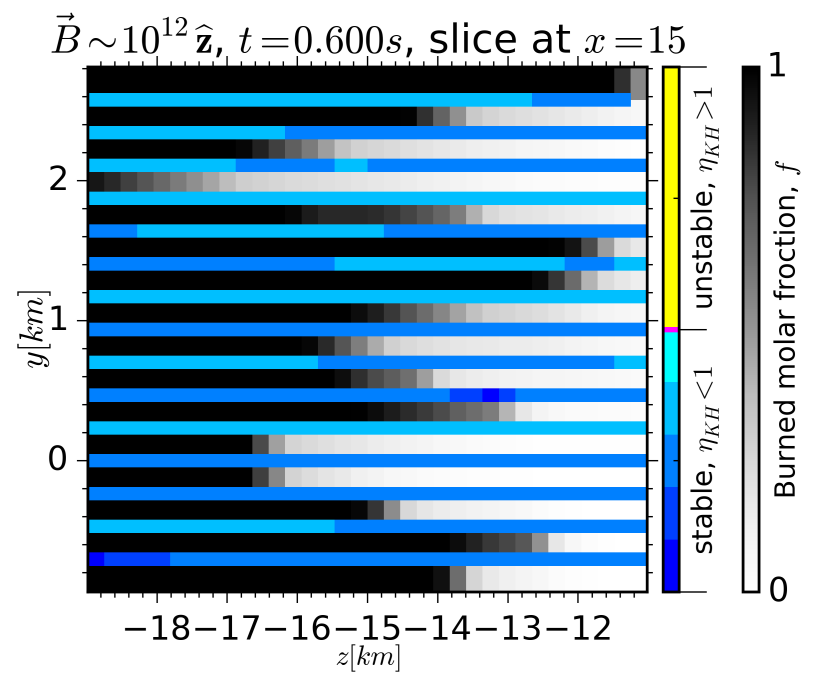

Fig. 9.- The "Kelvin-Helmholtz number" (Eq. C5 yellow-toblue scale) on the computational cells boundaries superimposed on the burned fraction (gray scale) for the Z12 model at $t=0.6 \mathrm{~s}$. Cold fuel sinks against the rising burned material, creating shear on the vertical cell boundaries. All lateral perturbations, i.e. the secondary Kelvin-Helmholtz instabilities, are suppressed by the magnetic field, sustaining the finger formations.

front becomes more laminar and less turbulent, and RT instabilities decrease. The effective front thickness also decreases by a factor of a few. One should ask whether medium fields are sufficient to bring significant change in the final outcome of the explosion, given that a Type Ia deflagration is estimated to only last about 2 seconds. The front speed and total burning rate tend to decrease as the field magnitude grows up to $10^{11} \mathrm{G}$ regardless of the field direction. Note that without steady state flows it is not meaningful to compare models one-to-one. However it is clear that the YZ12 and Z12 models have the fastest fronts and the effects of the direction of $B$ are stronger. When the longitudinal component of the field is strong, the burning is amplified and the front speed is highest and larger than the pure hydrodynamical case by a factor of 3 to 4 .

\subsection{Impact of Magnetic Fields}

These results suggest that strong magnetic fields might be missing from the picture of WD explosion physics painted by current 3D models. Suppression of large modes of RT instabilities in the early stages of the explosion is necessary to prevent large scale mixing until the transition to detonation. A second possible implication is related to the development of small scale structures which result in an effective increase in the 'surface' of the flame, which is the physical underpinning for a significant increase of the burning rate compared to a pure hydrodynamical front. If burning rate is amplified by magnetic fields it can lead to faster expansion and may freeze out the RT plumes in the unburned environment, consequently not allowing them rise to the surface, which also means less mixing.

As discussed in the introduction, the delayeddetonation model is currently the favorite for $M_{\mathrm{Ch}}$ explosions, but DDT remains unexplained. Possible processes based on the Zel'dovich mechanism are still under discussion. In order to increase the nuclear burning rate and form a detonation front, these require simultaneous mixing of burned and unburned matter on small scales over a significant volume. Possible physical causes for such mixing include mixing during a pulsational phase of the WD by crossing shock waves produced in the highly turbulent medium, shear flows and instabilities in the regime of distributed burning which are discussed in Poludnenko (2016). However, small scale fluctuations may be expected to prevent a DDT through mixing (Khokhlov et al. 1997, Niemeyer \& Woosley 1997). Another way to transition to detonation is by compression, when the speed of the burning front reaches the so called Chapman-Jougey limit. No solution for steady burning by deflagration exists above this limit, which for $\mathrm{C} / \mathrm{O}$ rich mixtures is about $40 \%$ of $v_{s}$ (Bruenn 1972). Based on 3D full-star simulations of deflagration fronts (e.g. Gamezo et al. 2003), pure deflagration fronts reach about 10$15 \%$ effective burning speed if full scale RT instabilities have developed over $\approx 2-3$ seconds. In all simulations, detonation in hydrodynamical models needs to be triggered "by hand". The RT stabilization effect of magnetic fields should additionally reduce the front surface, thus the front speed leading to a decreased likelyhood of DDT by either mechanism. Our results of small scale structure with an effective increase of the burning by factors of 3-5 in the YZ12 and Z12 models, may open an alternative mechanism for the DDT as a similar factor in the regime of distributed burning would bring the burning speed well above the Chapman-Jougey limit. Because the structure depends on the size and orientation of $B$, we must expect a wide variety and a dependence of the field morphology. A final answer is beyond this paper and will be addressed in full-star simulations in future works.

\subsection{Magnetic Field Growth and Saturation}

We have shown that $B$ fields larger than $10^{9 \ldots 10} \mathrm{G}$ will have a significant effect on the nuclear burning fronts under WD conditions, and may solve some of the current problems with $M_{\mathrm{Ch}}$ explosions and delayed-detonation models in particular. As discussed in the introduction, some WD have magnetic fields but, for most WDs, the $B$ fields are much smaller than those indicated by latetime observations of SNe Ia. Is it possible to produce high fields on relevant timescales prior to the explosion? Dynamos may be created during the accretion phase on time scales of $10^{6}$ years, or during the smoldering phase leading to the thermonuclear runaway on time scales of minutes (Hoeflich \& Stein 2002), or during the hydrodynamical phase of instabilities during the explosion on time scales of seconds or less. This leads us directly to $B$-field amplification in WDs and dynamo theories.

A seed magnetic field can be amplified by a dynamo operating in the convective zone of a differentially rotating star, including WDs (Parker 1979; Thomas et al. 1995: Brandenburg \& Subramanian 2005). In large scale dynamos, a toroidal field is produced by winding-up the poloidal component. The convective elements move upwards and downwards, perpendicular to the toroidal field lines, bending them and creating a new poloidal component (Parker 1979). Large scale dynamos grow with a typical timescale of the Alfvén times, $t_{A} \approx$ $R(4 \pi \rho)^{1 / 2} / B \approx 300 \mathrm{~s}$ (Parker 1979), which is comparable to the final final phase prior to the runaway. There- 
fore, this is not sufficient to increase the field by several orders of magnitude.

Alternatively, in the small scale dynamo, turbulence alone can amplify fields very quickly (Kazantsev 1968; Tayler 1973; Acheson 1978; Hawley et al.|1996; Spruit 2002; Brandenburg \& Subramanian 2005; Schekochihin \& Cowley 2007; Braithwaite 2009; Beresnyak et al. 2009; Duez et al. 2010b a). Within the core of a white dwarf close to $M_{\mathrm{Ch}}$, turbulence will undoubtedly play an enormous role. As the WD approaches $M_{C h}$, the polytropic index, $\gamma \rightarrow 4 / 3$, and the stability against radial motions disappears. Then kinetic energy will exhibit a Kolmogorov cascade, with energy distributed at all scales in a power law, $E=C k^{-5 / 3}$. This power law persists to the molecular viscous dissipation scale. Initially weak magnetic fields are stretched, twisted, and folded to increase the strength of the field. This mechanism will cause amplification until the kinetic and magnetic energies at a given scale are equal. In the kinematic phase, the magnetic energy is significantly below the kinetic energy. This occurs for large scales (small wave number) $k<k^{*}$, where the equipartition scale, $k^{*}$, is determined by the scale at which amplification is balanced by dissipation and transfer to higher scales. For small scales (large wave number), $k>k^{*}$, the magnetic field follows a Kolmorgorov cascade. The equipartition wavenumber, $k^{*}$, then decreases linearly with time (Schekochihin \& Cowley 2007, Beresnyak et al. 2009).

The details of this dynamo depend on the kinetic and magnetic dissipation scales. The kinematic viscosity and magnetic diffusivity have been found to be $\nu=3.13 \times 10^{-2} \mathrm{~cm}^{2} / \mathrm{s}$ and $\eta=5.6 \times 10^{-2} \mathrm{~cm}^{2} / \mathrm{s}$, respectively (Nandkumar \& Pethick 1984; Isern et al. 2017). With a typical velocity and length of $V \simeq 16 \mathrm{~km} / \mathrm{s}$ and $L \simeq 200 \mathrm{~km}$, respectively (e.g. Nonaka et al. 2012), we find kinetic and magnetic Reynolds numbers of $R e \equiv L V / \nu=10^{15}$ and $R m \equiv L V / \eta=5 \times 10^{14}$, respectively. We also find viscous and magnetic length scales of $\ell_{\nu}=L R e^{-3 / 4}=10^{-4} \mathrm{~cm}$ and $\ell_{\eta}=1.7 \times 10^{-4} \mathrm{~cm}$. Behavior of the turbulent dynamo depends on the magnetic Prandtl number, $P m=\nu / \eta=0.5$ for this system. In recent theoretical studies, (e.g. Schekochihin \& Cowley 2007, Beresnyak et al. 2009, Schober et al. 2012), it has been shown that the field can grow to energy equipartition at a given scale in an eddy turnover time at the smallest scale, essentially instantaneously, during the kinematic phase. Schober et al. (2012) estimate this growth rate for both large and small $\mathrm{Pm}$, finding that the growth rate $\Gamma=0.027 \mathrm{Rm}^{1 / 2} \mathrm{~V} / \mathrm{L}$, for low values of $P m$. Conservative estimates for the $L, V$ and $P m$ given above yield a growth rate of $5 \times 10^{4} \mathrm{~s}^{-1}$, or a doubling time of $2 \times 10^{-5} \mathrm{~s}$. Using length and velocity scales more appropriate for later phases of the WD (e.g. Hoeflich \& Stein 2002), $V \simeq 200 \mathrm{~km} / \mathrm{s}$ and $L \simeq 100 \mathrm{~km}$, we find $\Gamma=3 \times 10^{6} \mathrm{~s}^{-1}$.

It has been estimated (Schekochihin \& Cowley 2007) that the timescale for the equipartition length scale, $1 / k^{*}$, to reach the outer scale of the turbulence, $L$, is $t=L / U$. For our first estimate, this is $12 \mathrm{~s}$, while for our second it is $0.5 \mathrm{~s}$. To estimate the final level of magnetic field when the dynamo ceases to function, Schober et al. (2015) examine the feedback mechanism of Subramanian (1999). In this model, the magnetic diffusivity increases with magnetic energy. These authors find that in the small magnetic diffusivity limit as much as $40 \%$ of the total kinetic energy can be converted to magnetic energy. This is similar to numerical findings, for example Haugen et al. (2004) find 30\% of the energy is magnetic, Cho \& Vishniac (2000) who find 25\%, and (Beresnyak 2014) who finds $15 \%$. If the peak magnetic energy is some fraction, $f$, of the total,

$$
\frac{B^{2}}{8 \pi}=f \frac{1}{2} \rho V^{2},
$$

and $\rho=10^{9} \mathrm{~g} / \mathrm{cm}^{3}$ and $f=0.4$, we find a field of $10^{11} \mathrm{G}$ and $1.4 \times 10^{12} \mathrm{G}$ for the two conditions above. In a recent success of both laboratory plasma physics and theory, Tzeferacos et al. (2018) used the Omega laser facility at the University of Rochester to produce a $102 \mathrm{kG}$ magnetic field. They found a value of the ratio of magnetic energy to kinetic energy of $f=0.04$, which is somewhat lower than the numerical results, but will still result in a substantial magnetic field in a WD. Ultimately the details of the evolution depend on the details of the simulation, so full white dwarf simulations will be needed to specify the field for this specific system.

\subsection{Future Work}

We want to emphasize that this study presents only a first step to address the MHD problem for reactive fluids. Firm conclusions for an exploding WD require many more questions to be addressed. Here we will mention the limits of this study and questions which will be addressed in the near future by full-star models with our existing, more detailed nuclear networks. First, we treat the problem as if the WD was not expanding during the simulation - our gravitational acceleration is a constant instead of decreasing with time. Radial gradients in the gravitational acceleration, the initial density, and the initial pressure were neglected. Furthermore an evolved magnetic field would not be uniform, so the flame will encounter varying magnitude and direction as it advances. Moreover, the laminar diffusion speed will become directionally dependent. These effects will be studied in flux tubes using our Monte-Carlo transport coupled within a Particle in a Cell scheme. Finally, our nuclear network is too simple to carry out simulations in a distributed regime of burning should one want to include the detonation phase as well.

High resolution simulations of a full star are required in order to overcome the limitations of the flux tube results presented in this work. Other scientific questions we want to address in the future are: Are magnetic fields the missing physics in the current 3D models? In particular, do magnetic fields make pre-expansion of the WD possible; and if so, what is the mechanism - is it by suppressing the RT instabilities, by plume freeze-out on an accelerated background, or in some other way? What are observational signatures of the different field magnitudes and morphologies? Can they lead to different outcomes of the explosions, i.e. can different magnetic fields explain some of the diversity of SNe Ia?

\section{ACKNOWLEDGEMENTS}

C.A. Weatherford and B. Hristov were partially supported by the Department of Energy, National Nuclear 
Security Administration, under award DENA0002630, and the Nuclear Regulatory Commission, NRC-HQ-12G-27-0091. P. Hoeflich acknowledges support by the National Science Foundation (NSF) grant 1715133. This work used the Extreme Science and Engineering Discovery Environment (XSEDE), which is supported by the NSF grant ACI-1548562. Simulations were performed on Stampede and Maverick at the Texas Advanced Computing Center (https://www.tacc.utexas.edu), using XSEDE allocation TG-AST140008. Thank you to Braithwaite for helpful discussions on the dynamo theories and the formation of ultra-high magnetic fields in WD, to S. Shore for helpful discussions on MHD instabilities. Also thanks to Casey Mc Laughin and Prasad Maddumage, FSU Research Computing Center (https://rcc.fsu.edu), for help with technical problems. Visualization was done using yt (Turk et al.2011), matplotlib (Hunter 2007), and numpy (Van Der Walt et al. 2011). Thanks to Nathan Goldbaum and Matthew Turk for help on yt issues.

\section{APPENDIX \\ MASS AND MOLAR FRACTION DEFINITIONS AND IDENTITIES}

Here we define some quantities used in the text and give some conversions between them. Consider the fuel and product mixture, which we'll label with 1 and 2, respectively, to ease the notation. Obviously the partial mass densities add up to the total mass density:

$$
\rho=\rho_{1}+\rho_{2}
$$

We then define the mass, the molar, and the burned fractions, $\mathcal{X}, \mathcal{Y}$, and $f$ :

$$
\begin{gathered}
\mathcal{X}_{i} \equiv \rho_{i} / \rho, \quad \mathcal{Y}_{i} \equiv \mathcal{X}_{i} / \mathcal{A}_{i} \\
f \equiv \frac{\mathcal{Y}_{2}}{\mathcal{Y}_{1}+\mathcal{Y}_{2}}
\end{gathered}
$$

Clearly, the burned and the fuel fractions comprise the entirety of material, i.e. the fuel fraction equals to $1-f$, should one need it.

Using the definitions above it is easy to derive the following identities. The first two express the burned fraction, $f$, in terms of the total mass density, $\rho$, and one of the partial mass densities, $\rho_{1}$ or $\rho_{2}$. The following two equations show how to calculate the abundances, $\mathcal{Y}_{1,2}$, from the burned fraction, $f$.

$$
\begin{aligned}
& f=\frac{\mathcal{A}_{1}\left(\rho-\rho_{1}\right)}{\mathcal{A}_{1} \rho-\left(\mathcal{A}_{1}-\mathcal{A}_{2}\right) \rho_{1}}=\frac{\mathcal{A}_{1} \rho_{2}}{\mathcal{A}_{2} \rho+\left(\mathcal{A}_{1}-\mathcal{A}_{2}\right) \rho_{2}} \\
& \mathcal{Y}_{1}=\frac{1-f}{\mathcal{A}_{1}(1-f)+\mathcal{A}_{2} f}, \mathcal{Y}_{2}=\frac{f}{\mathcal{A}_{1}(1-f)+\mathcal{A}_{2} f}
\end{aligned}
$$

\section{REFERENCES}

Abel, T., Bryan, G. L., \& Norman, M. L. 2002, Science, 295, 93

Acheson, D. J. 1978, Royal Society of London Philosophical Transactions Series A, 289, 459

Alvear Terrero, D., Castillo García, M., Manreza Paret, D., Horvath, J. E., \& Pérez Martínez, A. 2015, Astronomische Nachrichten, 336, 851

Badenes, C., Borkowski, K., Hughes, J., Hwang, U., \& Bravo, E. 2006, ApJ, in press, astro-ph/0511140

Barbon, R., Benetti, S., Rosino, L., Cappellaro, E., \& Turatto, M. 1990, A\&A, 237, 79

Benz, W., Cameron, A. G. W., Press, W. H., \& Bowers, R. L. 1990, ApJ, 348, 647
Provided that we know $\rho$, from Equations A1 and A4 it follows that we only need the mass fraction and one of the partial mass densities to determine the other one. In it is the product mass density, $\rho_{2}$, that is being stored advected. The burning operator comes last in the time cycle and comprises the following steps: (i) find the burned fraction at the beginning of the cycle, using the econd of Eq. A4 (ii) evolve the burned fraction using a 27-point stencil for the Laplacian; (iii) update the density from Eq. A5 and (iv) update the energy from Eq. 12.

\section{LINEAR STABILITY THEORY}

We use analytic linear stability results from Chandrasekhar (1961). These come from linear stability analyses of the growth rate, $\Omega$, of normal perturbation modes, $\mathbf{k} \equiv k_{x} \widehat{\mathbf{i}}+k_{y} \widehat{\mathbf{j}}$ along the discontinuity interface, so that a perturbation is proportional to $\exp \left(i k_{x} x+i k_{y} y+\Omega t\right)$. Modes with such dependance on time are stable when $\Omega^{2}<0$.

We split the magnetic field into two terms, parallel and perpendicular to the gravity, i.e. $\mathbf{B} \equiv B_{z} \widehat{\mathbf{k}}+\mathbf{B}_{\perp}$, where $B_{z} \neq 0$ and $\mathbf{B}_{\perp} \equiv B_{x} \widehat{\mathbf{i}}+B_{y} \widehat{\mathbf{j}} \neq \mathbf{0}$, respectively. The dimensionless dispersion relation for $B_{z}$ is:

$$
\begin{array}{r}
\eta^{3}+2 \kappa\left(\alpha_{2}^{1 / 2}+\alpha_{1}^{1 / 2}\right) \eta^{2}+\kappa\left(2 \kappa+\alpha_{1}-\alpha_{2}\right) \eta- \\
-2 \kappa^{2}\left(\alpha_{2}^{1 / 2}-\alpha_{1}^{1 / 2}\right)=0
\end{array}
$$

where

$$
\begin{gathered}
\eta \equiv \frac{\Omega_{\|}}{\mathrm{g} / V_{A, z}}, \kappa \equiv \frac{k_{\|}}{\mathrm{g} / V_{A, z}^{2}}, \\
V_{A, z}^{2} \equiv \frac{B_{z}^{2}}{4 \pi\left(\rho_{2}+\rho_{1}\right)}, \alpha_{1,2} \equiv \frac{\rho_{1,2}}{\rho_{1}+\rho_{2}},
\end{gathered}
$$

and $\rho_{1}<\rho_{2}$. In this case no values of the parameters $B$ and $\rho_{1,2}$ yield stable modes.

We rewrite the dispersion relation for $\mathbf{B}_{\perp}$, for the modes parallel to the magnetic field, $\mathbf{k}_{\perp} \| \mathbf{B}_{\perp}$. Modes not parallel to $\mathbf{B}_{\perp}$ are "less" stable since the negative term should be multiplied by $\cos ^{2}\left(\mathbf{B}_{\perp}, \mathbf{k}_{\perp}\right)$.

$$
\Omega_{\perp}^{2}=g k_{\perp}\left(\frac{\rho_{2}-\rho_{1}}{\rho_{2}+\rho_{1}}-\frac{B_{\perp}^{2}}{2 \pi\left(\rho_{2}+\rho_{1}\right) g} k_{\perp}\right)
$$

Modes are stable when the right hand side of Eq. C4 is negative.

Finally we rewrite the condition for a mode to be Kelvin-Helmholtz stable as

$$
\eta_{K H} \equiv \frac{\alpha_{1} \alpha_{2} \Delta v_{z}}{2 V_{A}, z^{2}}<1 .
$$
our implementation of the new burning operator in Enzo 
Beresnyak, A. 2014, ApJ, 784, L20

Beresnyak, A., Jones, T. W., \& Lazarian, A. 2009, ApJ, 707, 1541

Braithwaite, J. 2009, MNRAS, 397, 763

Brandenburg, A., \& Subramanian, K. 2005, Phys. Rep., 417, 1

Bruenn, S. W. 1972, ApJ, 177, 459

Bryan, G. L., Machacek, M., Anninos, P., \& Norman, M. L. 1999, ApJ, 517, 13

Bryan, G. L., \& Norman, M. L. 1998, ApJ, 495, 80

Bryan, G. L., Norman, M. L., O'Shea, B. W., et al. 2014, ApJS, 211, 19

Chandrasekhar, S. 1961, Hydrodynamic and hydromagnetic stability

Cho, J., \& Vishniac, E. T. 2000, ApJ, 538, 217

Collins, D. C., Kritsuk, A. G., Padoan, P., et al. 2012, ApJ, 750, 13

Collins, D. C., Xu, H., Norman, M. L., Li, H., \& Li, S. 2010, ApJS, 186, 308

Diamond, T. R., Hoeflich, P., \& Gerardy, C. L. 2015, ApJ, 806, 107

Duez, M. D., Foucart, F., Kidder, L. E., Ott, C. D., \& Teukolsky, S. A. 2010a, Classical and Quantum Gravity, 27, 114106

Duez, V., Braithwaite, J., \& Mathis, S. 2010b, ApJ, 724, L34

Ferrario, L., de Martino, D., \& Gänsicke, B. T. 2015, Space Sci. Rev., 191, 111

Fesen, R. A., Hoeflich, P. A., Hamilton, A. J. S., et al. 2007, ApJ, 658,396

Fesen, R. A., Höflich, P. A., \& Hamilton, A. J. S. 2015, ApJ, 804, 140

Fisher, A., Branch, D., Nugent, P., \& Baron, E. 1997, ApJ, 481, L89

Foley, R. J., Challis, P. J., Chornock, R., et al. 2013, ApJ, 767, 57

Franzon, B., \& Schramm, S. 2017, ArXiv e-prints

Gamezo, V. N., Khokhlov, A. M., \& Oran, E. S. 2005, ApJ, 623, 337

Gamezo, V. N., Khokhlov, A. M., Oran, E. S., Ctchelkanova, A. Y., \& Rosenberg, R. O. 2003, Science, 299, 77

Gardiner, T. A., \& Stone, J. M. 2005, J. Comput. Phys, 205, 509

Ghezzi, C. R., de Gouveia Dal Pino, E. M., \& Horvath, J. E. 2001, ApJ, 548, L193

-. 2004, MNRAS, 348, 451

Giammichele, N., Bergeron, P., \& Dufour, P. 2012, ApJS, 199, 29

Haugen, N. E., Brandenburg, A., \& Dobler, W. 2004, Phys. Rev. E, 70, 016308

Hawley, J. F., Gammie, C. F., \& Balbus, S. A. 1996, ApJ, 464, 690

Hicks, E. P. 2015, ApJ, 803, 72

Hoeflich, P. 1995, ApJ, 443, 89

- 2006, Nuclear Physics A, 777, 579

Hoeflich, P., Gerardy, C. L., Fesen, R. A., \& Sakai, S. 2002, ApJ, 568,791

Hoeflich, P., Gerardy, C. L., Nomoto, K., et al. 2004, ApJ, 617, 1258

Hoeflich, P., \& Khokhlov, A. 1996, ApJ, 457, 500

Hoeflich, P., \& Stein, J. 2002, ApJ, 568, 779

Hoeflich, P., Hsiao, E. Y., Ashall, C., et al. 2017, ApJ, 846, 58

Höflich, P., Gerardy, C. L., Marion, H., \& Quimby, R. 2006, New A Rev., 50, 470

Howell, D. A., Hoeflich, P., Wang, L., \& Wheeler, J. C. 2001, ApJ, 556, 302

Howell, D. A., Sullivan, M., Nugent, P. E., et al. 2006, Nature, 443,308

Hoyle, F., \& Fowler, W. A. 1960, ApJ, 132, 565

Hristov, B., Collins, D. C., Hoeflich, P., \& Weatherford, C. 2016, in American Astronomical Society Meeting Abstracts, Vol. 227, American Astronomical Society Meeting Abstracts, 237.16

Hunter, J. D. 2007, Computing In Science \& Engineering, 9, 90

Iben, Jr., I., \& Tutukov, A. V. 1984, ApJS, 54, 335

Isern, J., García-Berro, E., Külebi, B., \& Lorén-Aguilar, P. 2017, ArXiv e-prints

Isern, J., Hernanz, M., \& José, J. 2011, in Lecture Notes in Physics, Berlin Springer Verlag, Vol. 812, Lecture Notes in Physics, Berlin Springer Verlag, ed. R. Diehl, D. H. Hartmann, \& N. Prantzos, 233-308

Ji, S., Fisher, R. T., García-Berro, E., et al. 2013, ApJ, 773, 136

Kawka, A., Vennes, S., Schmidt, G. D., Wickramasinghe, D. T., \& Koch, R. 2007, ApJ, 654, 499
Kazantsev, A. P. 1968, Soviet Journal of Experimental and Theoretical Physics, 26, 1031

Kepler, S. O., Pelisoli, I., Jordan, S., et al. 2013, MNRAS, 429 2934

Kepler, S. O., Pelisoli, I., Koester, D., et al. 2016, MNRAS, 455, 3413

Kerzendorf, W. E., McCully, C., Taubenberger, S., et al. 2017, MNRAS, 472, 2534

Khokhlov, A. M. 1995, ApJ, 449, 695

Khokhlov, A. M., Oran, E. S., \& Wheeler, J. C. 1997, ApJ, 478, 678

Kirshner, R. P., Jeffery, D. J., Leibundgut, B., et al. 1993, ApJ, 415,589

Kritsuk, A. G., Norman, M. L., Padoan, P., \& Wagner, R. 2007, ApJ, 665, 416

Kromer, M., Sim, S. A., Fink, M., et al. 2010, ApJ, 719, 1067

Kutsuna, M., \& Shigeyama, T. 2012, ApJ, 749, 51

Li, S., Li, H., \& Cen, R. 2008, ApJS, 174, 1

Li, W., Filippenko, A. V., Chornock, R., \& Jha, S. 2003, PASP, 115,844

Li, W., Bloom, J. S., Podsiadlowski, P., et al. 2011, Nature, 480, 348

Liebert, J., Bergeron, P., \& Holberg, J. B. 2003, AJ, 125, 348

Livne, E. 1990, ApJ, 354, L53

-. 1999, ApJ, 527, L97

Lorén-Aguilar, P., Isern, J., \& García-Berro, E. 2009, A\&A, 500 1193

Maeda, K., Benetti, S., Stritzinger, M., et al. 2010, Nature, 466, 82

Maeda, K., Leloudas, G., Taubenberger, S., et al. 2011, MNRAS, 413,3075

Marion, G. H., Hoeflich, P., Vacca, W. D., \& Wheeler, J. C. 2003, ApJ, 591, 316

Maund, J. R., Hoeflich, P. A., Patat, F., et al. 2010, ApJ, 725, L167

Mignone, A. 2007, J. Comput. Phys, 225, 1427

Milne, P., The, L.-S., \& Leising, M. 1999, ApJS, 124, 503

Motohara, K., Maeda, K., Gerardy, C. L., et al. 2006, ApJ, 652, L101

Nandkumar, R., \& Pethick, C. J. 1984, MNRAS, 209, 511

Niemeyer, J. C., \& Hillebrandt, W. 1995, ApJ, 452, 769

Niemeyer, J. C., \& Woosley, S. E. 1997, ApJ, 475, 740

Nomoto, K. 1982a, ApJ, 257, 780

- 1982b, ApJ, 253, 798

Nonaka, A., Aspden, A. J., Zingale, M., et al. 2012, ApJ, 745, 73

Norman, M. L., Reynolds, D. R., So, G. C., Harkness, R. P., \& Wise, J. H. 2015, ApJS, 216, 16

O'Shea, B. W., Wise, J. H., Xu, H., \& Norman, M. L. 2015, ApJ, 807, L12

Pakmor, R., Hachinger, S., Röpke, F. K., \& Hillebrandt, W. 2011, A\&A, 528, A117

Parker, E. N. 1979, Cosmical magnetic fields: Their origin and their activity (New York: Oxford University Press)

Patat, F., Hoeflich, P., Baade, D., et al. 2012, A\&A, 545, A7

Penney, R., \& Hoeflich, P. 2014, ApJ, 795, 84

Phillips, M. M. 1993, ApJ, 413, L105

Piersanti, L., Gagliardi, S., Iben, Jr., I., \& Tornambé, A. 2003, ApJ, 598, 1229

Plewa, T. 2007, ApJ, 657, 942

Poludnenko, A. 2016, Handbook of Supernovae, ed. P. Murdin (Springer, Berlin), 999

Poludnenko, A. Y., \& Oran, E. S. 2011, ArXiv e-prints

Potekhin, A. Y., \& Chabrier, G. 2012, A\&A, 538, A115

Pskovskii, I. P. 1977, Soviet Ast., 21, 675

Rasio, F. A., \& Shapiro, S. L. 1994, ApJ, 432, 242

Reinecke, M., Hillebrandt, W., \& Niemeyer, J. C. 1999, A\&A, 347,739

Reinsch, K., Euchner, F., Beuermann, K., Jordan, S., \& Gänsicke, B. T. 2005, in Astronomical Society of the Pacific Conference Series, Vol. 330, The Astrophysics of Cataclysmic Variables and Related Objects, ed. J.-M. Hameury \& J.-P. Lasota, 177 Remming, I. S., \& Khokhlov, A. M. 2014, ApJ, 794, 87 -. 2016, ApJ, 831, 162

Rest, A., Suntzeff, N. B., Olsen, K., et al. 2005, Nature, 438, 1132

Rest, A., Matheson, T., Blondin, S., et al. 2008, ApJ, 680, 1137

Röpke, F. K., Gieseler, M., Reinecke, M., Travaglio, C., \& Hillebrandt, W. 2006, A\&A, 453, 203 
Röpke, F. K., Kromer, M., Seitenzahl, I. R., et al. 2012, ApJ, 750, L19

Schekochihin, A. A., \& Cowley, S. C. 2007, Turbulence and Magnetic Fields in Astrophysical Plasmas, ed. S. Molokov, R. Moreau, \& H. K. Moffatt (Springer), 85

Schober, J., Schleicher, D., Bovino, S., \& Klessen, R. S. 2012, Phys. Rev. E, 86, 066412

Schober, J., Schleicher, D. R. G., Federrath, C., Bovino, S., \& Klessen, R. S. 2015, Phys. Rev. E, 92, 023010

Segretain, L., Chabrier, G., \& Mochkovitch, R. 1997, ApJ, 481, 355

Sion, E. M., Holberg, J. B., Oswalt, T. D., et al. 2014, AJ, 147, 129

Spruit, H. C. 2002, A\&A, 381, 923

Stehle, M., Mazzali, P. A., Benetti, S., \& Hillebrandt, W. 2005, MNRAS, 360, 1231

Stone, J. M., \& Gardiner, T. 2007a, Physics of Fluid Dynamics Letters, 19, 094104

-. 2007b, ApJ, 671, 1726

Stritzinger, M. D., Hsiao, E., Valenti, S., et al. 2014, A\&A, 561, A146

Subramanian, K. 1999, Physical Review Letters, 83, 2957

Sugimoto, D., \& Nomoto, K. 1980, Space Sci. Rev., 25, 155

Tanaka, M., Mazzali, P. A., Stanishev, V., et al. 2011, MNRAS, 410,1725
Tayler, R. J. 1973, MNRAS, 161, 365

Thomas, J. H., Markiel, J. A., \& van Horn, H. M. 1995, ApJ, 453, 403

Timmes, \& Woosley. 1992, ApJ, 396, 649

Turk, M. J., Smith, B. D., Oishi, J. S., et al. 2011, ApJS, 192, 9

Tzeferacos, P., Rigby, A., Bott, A. F. A., et al. 2018, Nature Communications, 9, 591

Van Der Walt, S., Colbert, S. C., \& Varoquaux, G. 2011, ArXiv e-prints

Wang, B., Chen, X., Meng, X., \& Han, Z. 2009a, ApJ, 701, 1540

Wang, B., Meng, X., Chen, X., \& Han, Z. 2009b, MNRAS, 395, 847

Webbink, R. F. 1984, ApJ, 277, 355

Whelan, J., \& Iben, I. J. 1973, ApJ, 186, 1007

Woosley, S. E., \& Kasen, D. 2011, ApJ, 734, 38

Woosley, S. E., \& Weaver, T. A. 1994, ApJ, 423, 371

Woosley, S. E., Weaver, T. A., \& Taam, R. E. 1980, in Texas Workshop on Type I Supernovae, ed. J. C. Wheeler, 96-112

Yang, Y., Wang, L., Baade, D., et al. 2017, ArXiv e-prints

Yoon, S.-C., Podsiadlowski, P., \& Rosswog, S. 2007, MNRAS, 380,933

Zhu, C., Pakmor, R., van Kerkwijk, M. H., \& Chang, P. 2015, ApJ, 806, L1 